\title{
Revised activity types for Markarian galaxies
}

\author{
A. M. Mickaelian, H. V. Abrahamyan, \\ G. S. Harutyunyan and G. M. Paronyan \\ Byurakan Astrophysical Observatory (BAO), Armenia \\ Email: aregmick@yahoo.com
}

\begin{abstract}
The sample of Markarian galaxies consists of 1515 UV-excess galaxies containing many active galaxies, both AGN and Starburst (SB). Several catalogs of Markarian galaxies have been published; however activity types are based on old spectroscopic data. The SDSS spectroscopy and some other recent spectral observations allow classify or re-classify many of Markarian galaxies, altogether we have retrieved and studied 779 SDSS and 300 other spectra. Out of 779 SDSS spectra, we have classified 533 HII, 31 Composites, 12 LINERs, 4 S2.0, 5 S1.9, 8 S1.8, 5 NLS1.5, 11 S1.5, 8 NLS1.2, 21 S1.2, 4 NLS1, 4 S1.0, 2 QSO, 11 AGN (without an exact class), $52 \mathrm{Em}$ (HII or AGN), $65 \mathrm{Abs,}$ and 3 Stars. On the other hand, the galaxies are being classified depending on the fact in which wavelength range they have been observed and studied. E.g. some Sy2 type galaxies turn to be Sy1 when classified in IR. Many hidden AGN (in X-ray and IR) appear to be normal galaxies in optical range. So for better understanding, IR spectra are necessary as well.
\end{abstract}

Keywords. Markarian galaxies, spectroscopy, AGN, Seyfert galaxies

Markarian survey (Markarian et al. 1989) led to discovery of 1515 UV-excess (Markarian) galaxies, including many active ones, both AGN and Starburst (SB) galaxies. Several catalogues of Markarian galaxies have been published (Mazzarella \& Balzano 1986; Markarian et al. 1989, 1997; Bicay et al. 1995; Petrosian et al. 2007), however in all these catalogues, activity types are based on old (mostly poor quality) spectroscopic data. 292 Markarian galaxies (296 with components) appear in the Catalogue of QSOs and Active Galaxies (version 13, Veron-Cetty \& Veron 2010, hereafter VCV-13), where redshifts, activity types, absolute magnitudes and some other data are given. There are 3 blazars, 5 QSOs, 65 Sy1.0, 21 NLS1, 13 S1.2, 42 Sy1.5, 12 Sy1.8, 10 Sy1.9, 46 Sy2.0, 11 LINERs, $15 \mathrm{Sy}$ (without a definite type), $51 \mathrm{HII}$, and 2 AGN without any classification.

The SDSS (Ahn et al. 2013) spectroscopy and some other recent spectral observations allow classify or re-classify many of Markarian galaxies. We have retrieved and studied 779 SDSS DR7, DR8, and DR9 and 300 other spectra (SDSS area is less than FBS and hence not all Markarian galaxies are in SDSS). Fig. 1 shows SDSS spectra for two Markarian galaxies: Mrk 142 (NLS1) and Mrk 1469 (Sy1.8). We have identified all spectral lines, measured their FWHM, intensities, and equivalent widths (EW) and built diagnostic diagrams using line ratios $[\mathrm{OIII}] / \mathrm{H} \beta$ vs. $[\mathrm{OI}] / \mathrm{H} \alpha$ vs. and $[\mathrm{OIII}] / \mathrm{H} \beta$ vs. $[\mathrm{NII}] / \mathrm{H} \alpha$ (Veilleux \& Osterbrock 1987). As not all data can be measured with high accuracy, we also use eye classification to compare with the results from diagnostic diagrams. We have tried to classify all objects using the fine details, including the intensity of FeII lines to distinguish Narrow Line Seyferts (NLS). We have introduced subclasses of NLS for each subtype of Seyferts: S1.0, S1.2, and S1.5 (later subtypes typically do not show such features).

We give in Table 1 the average physical properties (redshifts and absolute magnitudes) for various activity types of Markarian galaxies. NLS are somewhat intermediate between high-luminosity (S1.0, S1.2, S1.5) and low-luminosity (S1.8, S1.9, S2.0) Seyferts. 

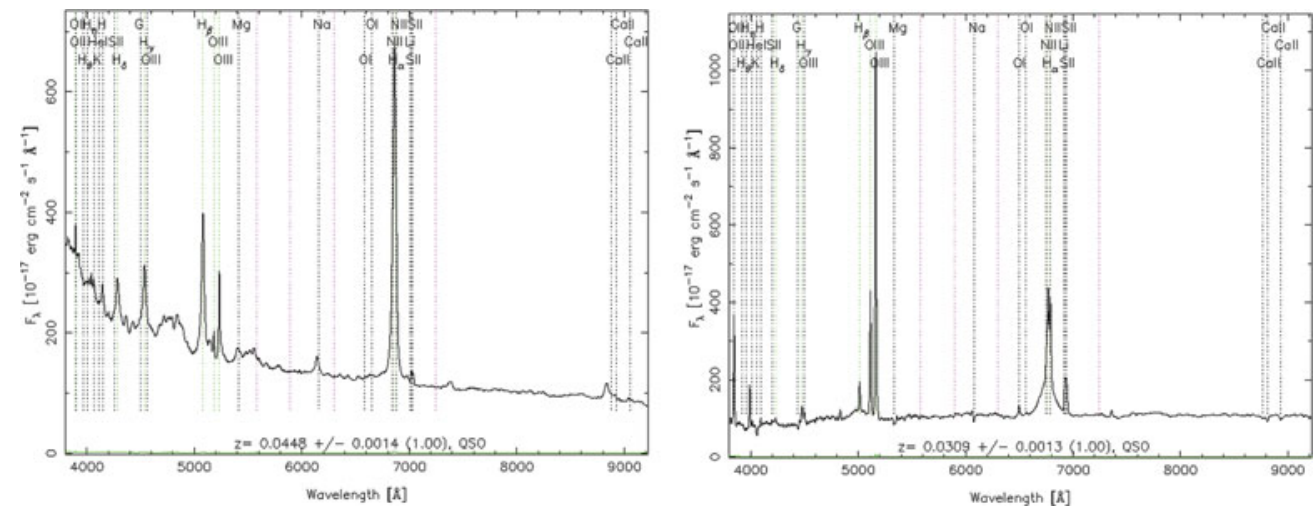

Figure 1. SDSS spectra for two Markarian galaxies: Mrk 142 (left, NLS1) and Mrk 1469 (right, S1.8).

Table 1. Redshifts and absolute magnitudes for various activity types of Markarian galaxies.

\begin{tabular}{|c|c|c|c|c|}
\hline Activity type & M range & M average & Z range & Z average \\
\hline HII & $-23.04 \ldots-13.58$ & -19.75 & $0.00347-0.09480$ & 0.02526 \\
LINER & $-22.85 \ldots-19.08$ & -20.74 & $0.00589-0.04866$ & 0.02726 \\
Composite & $-22.89 \ldots-18.21$ & -21.15 & $0.00510-0.06614$ & 0.03051 \\
S1.8, S1.9, S2.0 & $-22.72 \ldots-20.41$ & -21.64 & $0.01415-0.21028$ & 0.04901 \\
NLS1, NLS1.2, NLS1.5 & $-23.53 \ldots-20.04$ & -21.63 & $0.02311-0.16349$ & 0.06047 \\
S1.0, S1.2, S1.5 & $-22.92 \ldots-19.62$ & -21.72 & $0.01627-0.18634$ & 0.05798 \\
QSO & $-27.70 \ldots-27.42$ & -27.56 & $1.75707-1.90401$ & 1.83054 \\
\hline
\end{tabular}

Out of 779 SDSS spectra, we have classified 533 HII regions, 31 Composites (LINER/HII, S/HII, S/LINER or S/HII/LINER), 12 LINERs, 4 S2.0, 5 S1.9, 8 S1.8, 5 NLS1.5, 11 S1.5, 8 NLS1.2, 21 S1.2, 4 NLS1, 4 S1.0, 2 QSO, 11 AGN (without an exact class), 52 Emission-line galaxies (HII or AGN), 65 Absorption-line galaxies, and 3 Stars. On the other hand, the galaxies are being classified depending on the fact in which wavelength range they have been observed and studied. E.g. some Sy2 type galaxies turn to be Sy1 when classified in IR. Many hidden AGN (in X-ray and IR) appear to be normal galaxies in optical range. So for better understanding, IR spectra are necessary as well, and we plan to extract and study Spitzer spectra too.

Thus, we have revised and refined the activity types for 779 Markarian galaxies, especially the subclasses of Seyferts. Many S1 were re-classified to S1.2 or later subtypes. There appeared especially many NLS and intermediate objects, where the permitted lines have intermediate widths and weak FeII lines are observed. However, the total fraction of AGN among all Markarian galaxies did not change much; now 16.17\% are AGN compared to $15.91 \%$ by former classifications.

\section{References}

Ahn, C. P., Alexandroff, R., Allende Prieto, C., et al. 2013, ApJS, in press.

Bicay M. D., Kojoian G., Seal J., et al. 1995, ApJS 98, 369

Markarian, B. E., Lipovetski, V. A., Stepanian, J. A., et al. 1989, Com. SAO 62, 5

Markarian B. E., Lipovetsky V. A., Stepanian J. A., et al. 1997, Vizier Catalogue VII/172

Mazzarella J. M. \& Balzano V. A. 1986, ApJS 62, 751

Petrosian A., McLean B., Allen R. J., \& MacKenty J. W. 2007, ApJS 170, 33

Veilleux, S. \& Osterbrock, D. E. 1987, ApJS 63, 295

Veron-Cetty M. P. \& Veron P. 2010, A\&A 518, A10 